\title{
Inversion of polarimetric data from eclipsing binaries
}

\author{
I.J. Coleman, N. Gray, and J.F.L. Simmons \\ Department of Physics and Astronomy, University of Glasgow, Glasgow G12 8QQ, Scotland, UK
}

Received February 6, 1997; accepted February 18, 1998

\begin{abstract}
We describe a method for determining the limb polarization and limb darkening of stars in eclipsing binary systems, by inverting photometric and polarimetric light curves.

Because of the ill-conditioning of the problem, we use the Backus-Gilbert method to control the resolution and stability of the recovered solution, and to make quantitative estimates of the maximum accuracy possible. Using this method we confirm that the limb polarization can indeed be recovered, and demonstrate this with simulated data, thus determining the level of observational accuracy required to achieve a given accuracy of reconstruction. This allows us to set out an optimal observational strategy, and to critcally assess the claimed detection of limb polarization in the Algol system.

The use of polarization in stars has been proposed as a diagnostic tool in microlensing surveys by Simmons et al. (1995), and we discuss the extension of this work to the case of microlensing of extended sources.
\end{abstract}

Key words: polarization - methods: data analysis binaries: eclipsing — gravitational lensing

\section{Introduction}

Scattered light emerging from a stellar atmosphere is expected to be partially linearly polarized. This effect should be greatest at the limb of the star, with a pure electron scattering atmosphere giving a limb polarization of $11.7 \%$ (Chandrasekhar 1946, 1950). Less idealised calculations (Collins \& Buergher 1974) suggest a lower degree of limb polarization, around $2 \%$ for early-type stars, though this figure is rather sensitive to the ionization state of the outermost atmospheric layers. Clearly a spherically symmetric star will exhibit no net polarization, but if the symmetry is broken by an eclipse the limb polarization should

Send offprint requests to: N. Gray in principle be detectable. Theoretical polarimetric light curves for this situation have been calculated by Landi Degl'Innocenti et al. (1988).

The first detection of this "Chandrasekhar effect" was in the Algol system (Kemp et al. 1983). This data was analysed by Wilson \& Liou (1993), but the complexity of the system and the amount of modelling involved in the analysis prevented them from making any reliable estimate of the limb polarization.

The inversion of polarimetric light curves from eclipsing binary stars should allow the limb polarization of the eclipsed star to be measured (and the photometric light curve should similarly give the limb darkening). In fact this inverse problem is highly ill-conditioned, and relating observations to stellar atmosphere models is therefore far from straightforward. In this paper we investigate the practical feasibility of determining of limb polarization by this method.

This allows us to address three closely related issues:

1. We develop a method of obtaining the polarization at a point on the stellar disc, and of estimating the error on this value. This is based on the Backus-Gilbert inversion technique.

2. We determine the maximium accuracy possible in determining limb polarization, given a number of data points and a noise level.

3. Thus, we are able to put forward an observational strategy which should allow the best measurement of limb polarization.

In Sect. 2 we give a brief overview of the problem. We set out the formalism of the Backus-Gilbert method in Sect. 3, and discuss its suitability for the problem at hand. Section 4 contains the calculations for the specific case of eclipsing binary stars, and Sect. 5 presents the results of the inversion scheme when applied to simulated data, and the conclusions that can be drawn from these. Section 6 considers a simplified analogue of the Algol system, comparing the theoretical polarization profile with the best resolution current measurements can achieve. 


\section{An overview of the problem}

The radiation field across the stellar disk can be described by the stokes parameters, which give the unpolarized and polarized intensity. These are usually denoted by $I, Q$, $U$, and $V$ (these parameters are discussed more fully in Clarke \& Grainger 1971). Here we shall take the circular polarization $V$ to be zero. $U$ and $Q$ give the state of linear polarization of the radiation. $Q$ is obtained by measuring the difference in intensity in two perpendicular directions, and $U$ by measuring the difference when the polarimiter is rotated through $45^{\circ}$. The degree of linear polarization may simply be written as $\left(Q^{2}+U^{2}\right)^{1 / 2} / I$ and the position angle as $\frac{1}{2} \arctan U / Q$. Under rotation of axes by $\psi, Q$ and $U$ simply transform as $U_{\text {new }}=U_{\text {old }} \cos 2 \psi-Q_{\text {old }} \sin 2 \psi$ and $Q_{\text {new }}=U_{\text {old }} \sin 2 \psi+Q_{\text {old }} \cos 2 \psi$. I is invariant (as is $V)$.

We will assume that the light emerging from the stellar disk is partially linearly polarized perpendicular to the radial direction. One can calculate the total flux from the eclipsed star by integrating the intensity $I$ over the visible part of the disc. Similarly, to obtain the total polarized flux one simply integrates the Stokes parameter $Q$ over the same part of the disc, introducing a rotation factor to transform the polarized intensity at each point into the appropriate reference frame.

$$
\begin{aligned}
& F_{\mathrm{I}}(t)=\frac{1}{R^{2}} \iint_{\text {disc }} I(r) A(r, \phi, t) r \mathrm{~d} r \mathrm{~d} \phi \\
& F_{\mathrm{Q}}(t)=\frac{1}{R^{2}} \iint_{\text {disc }} Q(r) A(r, \phi, t) \cos 2 \phi r \mathrm{~d} r \mathrm{~d} \phi \\
& F_{\mathrm{U}}(t)=\frac{1}{R^{2}} \iint_{\text {disc }} Q(r) A(r, \phi, t) \sin 2 \phi r \mathrm{~d} r \mathrm{~d} \phi
\end{aligned}
$$

where $(r, \phi)$ are polar coordinates on the stellar disk, $R$ is the distance from the observer to the star, and $A(r, \phi, t)$ is zero for an occulted point on the disk, 1 otherwise.

In this paper we address the problem of extracting $I(r)$ and $Q(r)$ from measurements of $F_{\mathrm{I}}, F_{\mathrm{Q}}$ and $F_{\mathrm{U}}$ during an eclipse. One might attempt to do this by modelling $I(r)$ and $Q(r)$ for the stellar atmosphere, performing the integrals above and using the data to fit the free parameters in the stellar atmosphere model. But the problem must be treated more carefully. It is a general property of inverse problems, such as the present case, that a very large range of source models are consistent with a given data set. This is essentially due to the smoothing properties of the kernel (here, the occultation function $A$ ). Consequently, a forward-modelling approach can give apparently accurate, but actually highly misleading, results. A thorough treatment of inverse problems in astronomy can be found in Craig \& Brown (1986); here we will simply state that meaningful results can only be obtained by controlling the instabilities caused by discrete, noisy data. We have used the Backus-Gilbert inversion technique to achieve this control. The principal advantage of the
Backus-Gilbert method from our point of view is that it allows us a qualitative understanding of, and thus an explicit quantitative control over, the compromise between bias and stability in our inversion.

\section{The Backus-Gilbert method}

The Backus-Gilbert method is one of a family of methods for attacking inverse problems. There are several general introductions to the method (Parker 1977, gives an excellent review, and Loredo \& Epstein 1989, gives an example in an astrophysical context) - we give a minimal introduction here, partly in order to fix our notation. There is a more formal discussion of the method in an appendix, and a thorough treatment can be found in Backus \& Gilbert (1970).

We wish to recover an underlying function $u(r)$, in this case, either an intensity, or the run of polarization with radius; the parameter $r$ here is the projected radial distance from the centre of the eclipsed star's disk, in units where the star's radius is $r=1$. We cannot measure this underlying function directly, but instead measure an integral of it, $F(s)$, which might be the observed luminosity or polarisation of the eclipsed star. This is related to the underlying function through

$$
F(\boldsymbol{s})=\int_{0}^{1} u(r) K(r ; \boldsymbol{s}) \mathrm{d} r
$$

where $s$ is the vector between the centres of the star and its occultor, and the kernel $K(r ; s)$ can be calculated a priori. The set of observations that we make, $f_{i} \equiv F\left(s_{i}\right)$, is therefore related to this underlying function by

$f_{i}=\int_{0}^{1} u(r) K_{i}(r) \mathrm{d} r+n_{i}$,

where $K_{i}(r) \equiv K\left(r ; \boldsymbol{s}_{i}\right)$ and $n_{i}$ is a random admixture of noise. From these measurements we wish to produce an estimator $\hat{u}(r)$ of the underlying function $u(r)$.

For the Backus-Gilbert method, we suppose that the mean of the estimator and the underlying function are related by an averaging kernel $\Delta\left(r, r^{\prime}\right)$, through

$E(\hat{u}(r))=\int_{0}^{1} \Delta\left(r, r^{\prime}\right) u\left(r^{\prime}\right) \mathrm{d} r^{\prime}$

Since we do not know the underlying function, the averaging kernel is of no use to us directly; however we can study its properties, and use our data $f_{i}$ in such a way as to optimise those properties, and so minimise the dependence of the estimate $\hat{u}(r)$ on the underlying function and the noise. It is clear from Eq. (6) that $\hat{u}(r)=u(r)$ if $\Delta\left(r, r^{\prime}\right)$ is the Dirac delta function. However, such a solution is highly sensitive to noise in the data and hence very unstable. We attain stability by increasing the width of $\Delta\left(r, r^{\prime}\right)$, and hence smoothing the recovered value over a wider region of the source - that is, we minimise the width of $\Delta\left(r, r^{\prime}\right)$ subject to the conflicting demand that 
the resolution of $\Delta\left(r, r^{\prime}\right)$ is sufficient for the problem at hand, in a sense we shall make more precise below.

We relate our data $f_{i}$ to our estimator $\hat{u}(r)$ through a set of response kernels $q_{i}(r)$, which produce an estimate of the underlying function through

$\hat{u}(r)=\sum_{i} q_{i}(r) f_{i}$.

If we substitute Eq. (5) into Eq. (7), and assume $E\left(\sum_{i} q_{i}(r) n_{i}\right)=0$, then we can compare with Eq. (6), and obtain

$\Delta\left(r, r^{\prime}\right)=\sum_{i} q_{i}(r) K_{i}\left(r^{\prime}\right)$

This allows us to form some measure of the width of $\Delta\left(r, r^{\prime}\right)$ such as

$$
\begin{aligned}
\mathcal{A} & \equiv \int\left(r-r^{\prime}\right)^{2}\left[\Delta\left(r, r^{\prime}\right)\right]^{2} \mathrm{~d} r^{\prime}, \\
& =\sum_{i j} q_{i}(r) W_{i j}(r) q_{j}(r) \\
& =\boldsymbol{q}(r)^{T} \boldsymbol{W}(r) \boldsymbol{q}(r),
\end{aligned}
$$

which depends on $q_{i}$, and depends on $K_{i}$ through the definition

$$
W_{i j}(r) \equiv \int_{0}^{1}\left(r^{\prime}-r\right)^{2} K_{i}\left(r^{\prime}\right) K_{j}\left(r^{\prime}\right) \mathrm{d} r^{\prime} .
$$

This is the standard definition of the width; others are reasonable, and may be preferable in different circumstances.

Again assuming that $E(\boldsymbol{q} \cdot \boldsymbol{n})=0$, we can also form a measure of the stability of Eq. (7)

$\mathcal{B} \equiv \operatorname{Var} \hat{u}(r)=\boldsymbol{q}(r)^{T} \boldsymbol{S} \boldsymbol{q}(r)$,

which depends on $q_{i}$ and the noise covariance matrix $S_{i j} \equiv$ $E\left(n_{i} n_{j}\right)$. In our simulations below, we take the $n_{i}$ to be independent and Gaussian with standard deviation $\sigma$; in this case, $S_{i j}=\delta_{i j} \sigma^{2}$, and our assumption $E(\boldsymbol{q} \cdot \boldsymbol{n})=0$ is true.

Finally, the demand that $\Delta\left(r, r^{\prime}\right)$ in Eq. (6) have unit area, leads to the constraint

$\boldsymbol{q}(r) \cdot \boldsymbol{R}=1$,

where $R_{i} \equiv \int K_{i}(r) \mathrm{d} r$.

The Backus-Gilbert method consists of finding those $q_{i}(r)$ which minimise

$$
\begin{aligned}
\mathcal{A}+\lambda \mathcal{B} & =\boldsymbol{q}(r) \cdot[\boldsymbol{W}(r)+\lambda \boldsymbol{S}] \cdot \boldsymbol{q}(r) \\
& =\int\left(r-r^{\prime}\right)^{2}\left[\Delta\left(r, r^{\prime}\right)\right]^{2} \mathrm{~d} r^{\prime}+\lambda \operatorname{Var} \hat{u}(r),
\end{aligned}
$$

for some selected parameter $\lambda$, subject to the constraint $\boldsymbol{q} \cdot \boldsymbol{R}=1$. The minimisation problem has explicit analytic solutions

$$
\boldsymbol{q}_{\lambda}(r)=\frac{[\boldsymbol{W}(r)+\lambda \boldsymbol{S}]^{-1} \cdot \boldsymbol{R}}{\boldsymbol{R} \cdot[\boldsymbol{W}(r)+\lambda \boldsymbol{S}]^{-1} \cdot \boldsymbol{R}}
$$

in terms of the parameter $\lambda$, and these different solutions, when combined with the data $f_{i}$ using Eq. (7), give different estimators $\hat{u}_{\lambda}(r)$. The nature of the trade-off in the minimisation is clear: in order to improve the stability of the recovery, we choose a $\lambda$ which makes $\Delta\left(r, r^{\prime}\right)$ broader, and so generate response kernels $q_{i}$ which extend the weighted average over a greater number of the data points $f_{i}$. The cost of this is that the estimate of the recovered point will be biased by the inclusion of the extra data, and this will be more marked when the underlying function is rapidly varying.

The first important point about the Backus-Gilbert method is that the parameter $\lambda$ allows us to adjudicate between the conflicting demands of minimising the width of the kernel $\Delta\left(r, r^{\prime}\right)$ and minimising the sensitivity of the recovered value (which is a realisation of the statistical variable $\left.\hat{u}_{\lambda}(r)\right)$ to the measurement noise, and that this adjudication can be done prior to any data being collected, based only on the characteristics of the kernel $K(r ; s)$ and the noise.

Secondly, we must emphasise that the $\boldsymbol{q}_{\lambda}(r)$ we obtain gives us, through Eq. (7), a single point in the recovered function, $\hat{u}_{\lambda}(r)$. This means that in this simplest version of the Backus-Gilbert method we must perform the inversion for each value of $r$ for which we wish to find $\hat{u}_{\lambda}(r)$. Since the calculation of the coefficients $\boldsymbol{q}_{\lambda}(r)$ involves a matrix inversion, which is an $n^{3}$ procedure, it can be computationally expensive, but this limitation is acceptable in our particular case, as we only wish to recover the polarisations at the limb, $r=1$. This feature has the compensation that we can if necessary select a different optimal value of $\lambda$ for each recovered point.

\section{Kernels for the eclipsing case}

In this section we derive the kernels for the case when one star is partially eclipsed by another (a future paper (Coleman et al. in preparation) will discuss the case of a gravitational lens). The geometry of this situation is as shown in Fig. 1. We can take the occultor to be opaque, so that its "transfer function" $A_{0}\left(r_{0}\right)$, where $r_{0}$ is the (projected) radius from the centre of the occultor, is

$A_{0}\left(r_{0}\right)=\left\{\begin{array}{ll}0 & \text { for } r_{0}<\rho \\ 1 & \text { for } r_{0}>\rho\end{array}\right.$.

This makes $A(r, \chi ; s)$, the kernel in terms of $r$, a function of $\psi(r ; s)$. In terms of $i(r)$, the intensity of the star as a function of (projected) radius, the total flux from the eclipsed star is

$F_{\mathrm{I}}(s)=\int_{\text {area }} i(r) A(r, \chi ; s)\left(\mathrm{d}^{2} \boldsymbol{r}=r \mathrm{~d} r \mathrm{~d} \chi\right)$.

Thus define $\tilde{A}(r ; s)=r \int_{0}^{2 \pi} A(r, \chi ; s) \mathrm{d} \chi$, so that

$F_{\mathrm{I}}(s)=\int_{0}^{1} i(r) \tilde{A}(r ; s) \mathrm{d} r$,

putting Eq. (15) into the form of Eq. (4).

It is easy to see that $\tilde{A}(r ; s)=r \int_{0}^{2 \pi-\psi(s)} 1 \mathrm{~d} \chi$. Writing $\gamma \equiv \cos [\psi(r ; s) / 2]$ we find that

$\gamma=\left\{\begin{array}{lr}1 & , \quad 1<\bar{\gamma} \\ \bar{\gamma} \equiv\left(r^{2}+s^{2}-\rho^{2}\right) / 2 r s & ,-1 \leq \bar{\gamma} \leq 1 \\ -1 & , \quad \bar{\gamma}<-1\end{array}\right.$ 


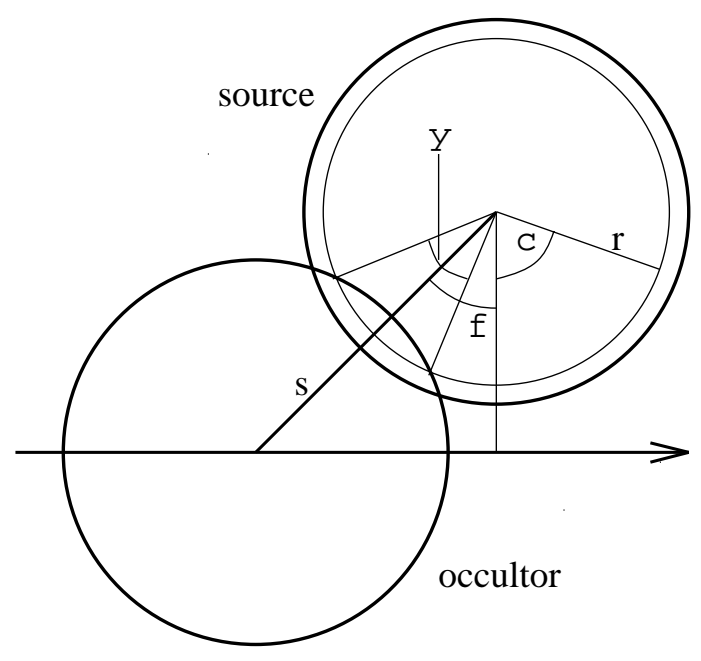

Fig. 1. The eclipsed star has radius 1 , and the occultor radius $\rho$. $(r, \chi)$ are polar coordinates in the projected plane of the source, and $(s(t), \phi(t))$ are the coordinates of the centre of the occultor; both $\chi$ and $\phi$ are taken from the radius which touches the point of closest approach. When the centres of the two stars are a distance $s$ apart, the occultor cuts off an angle $\psi(r ; s, \rho)$ of an annulus of radius $r$, centred on the eclipsed star

We can now write

$\tilde{A}(r ; s)= \begin{cases}2 r(\pi-\psi / 2) & \\ =2 r \arccos (-\gamma) & ,|s-\rho| \leq r \leq s+\rho \\ 0 & , r<-(s-\rho) \\ 2 \pi r & , \text { otherwise }\end{cases}$

defined for $r \geq 0$.

The calculation is a little more intricate for the Stokes parameters. The light from each point on the star's disk must be linearly polarized in the tangential direction. Using the angle $\chi$ defined in Fig. 1, the Stokes parameters must therefore be $F_{\mathrm{U}}(r, \chi)=-P(r) \sin 2 \chi$ and $F_{\mathrm{Q}}(r, \chi)=-P(r) \cos 2 \chi$, for some function $P(r)$ which we wish to recover (note that we use the unnormalised Stokes parameters, since the normalised ones have contributions to the noise from the intensity as well as the polarization measurements). Defining

$\tilde{A}_{\mathrm{Q}}(r ; s, \phi)=-r \int_{0}^{2 \pi} \cos 2 \chi A(r, \chi ; s, \phi) \mathrm{d} \chi$,

we therefore find that the total polarized flux in the $Q$ direction, measured when the centres are a distance $s$ apart, is

$F_{\mathrm{Q}}(s(t), \phi(t))=\int_{0}^{1} P(r) \tilde{A}_{\mathrm{Q}}(r ; s, \phi) \mathrm{d} r$,

and similarly for $A_{\mathrm{U}}(r, \chi ; s, \phi)$ and $F_{\mathrm{U}}(s, \phi)$. This is now in the form of Eq. (4). Setting $l=s \cos \phi$, we can thus see that

$$
\begin{aligned}
\tilde{A}_{\mathrm{Q}}(r ; s, \phi) & =r \cos 2 \phi(t) \sin \psi(r ; s(t)) \\
& =2 r\left(2 \frac{l^{2}}{s^{2}}-1\right) \gamma \sqrt{1-\gamma^{2}}
\end{aligned}
$$

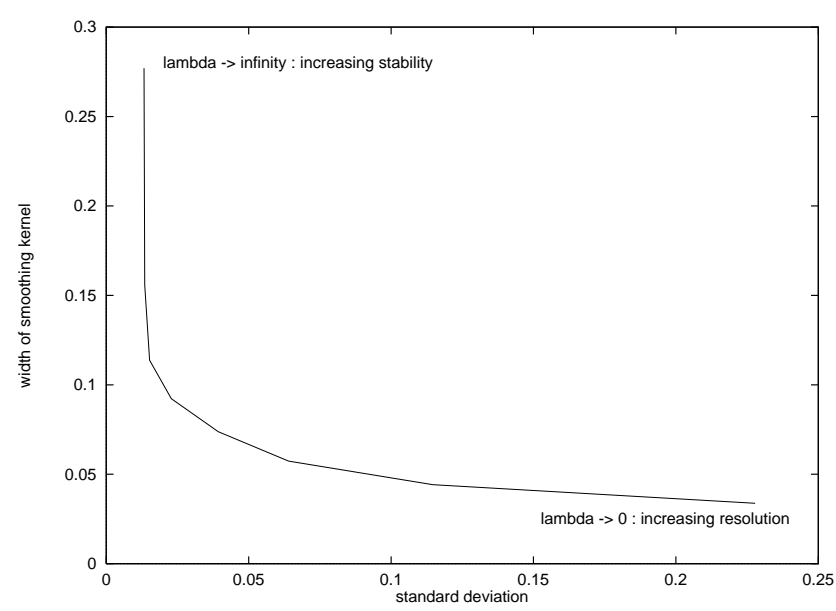

Fig. 2. An example of a trade-off curve for the Backus-Gilbert inversion scheme

$$
\begin{aligned}
\tilde{A}_{\mathrm{U}}(r ; s, \phi) & =r \sin 2 \phi \sin \psi \\
& =4 r \frac{l}{s}\left(1-\frac{l^{2}}{s^{2}}\right)^{\frac{1}{2}} \gamma \sqrt{1-\gamma^{2}}
\end{aligned}
$$

These kernels are broad and smooth, hence the illconditioning of the inverse problem.

\section{Polarization data inversion}

One strength of the Backus-Gilbert method is that we can do a lot of the analysis before we have any data. This gives us an understanding of the limitations of our analysis, and allows us to pick an optimal value for the smoothing parameter $\lambda$.

\subsection{Theoretical limits on polarization inversions}

The Backus-Gilbert method is one of many inverse problem methods in which one functional of the recovered solution, $\mathcal{A}$, is minimised subject to regulation by another functional $\mathcal{B}$. In this case, functional $\mathcal{A}$ is the width of the averaging kernel, and this is regulated by the variance of the estimate. In other inverse problem methods, the functional $\mathcal{A}$ is some measure of the goodness of fit between the data and the forward problem, such as a $\chi^{2}$, regulated by the demand that the solution be smooth, or that some non-linear functional of the solution, such as its negentropy, be minimised.

The Backus-Gilbert scheme produces a onedimensional family of solutions, parametrised by the smoothing parameter $\lambda$. We can represent this as a solution curve on a graph of standard deviation against of the recovered $\hat{u}$ against the chosen measure of the width of the resolution function: the resolution is inversely related to the width, so such a curve illustrates the trade-off between accuracy and bias in the recovered solution. 


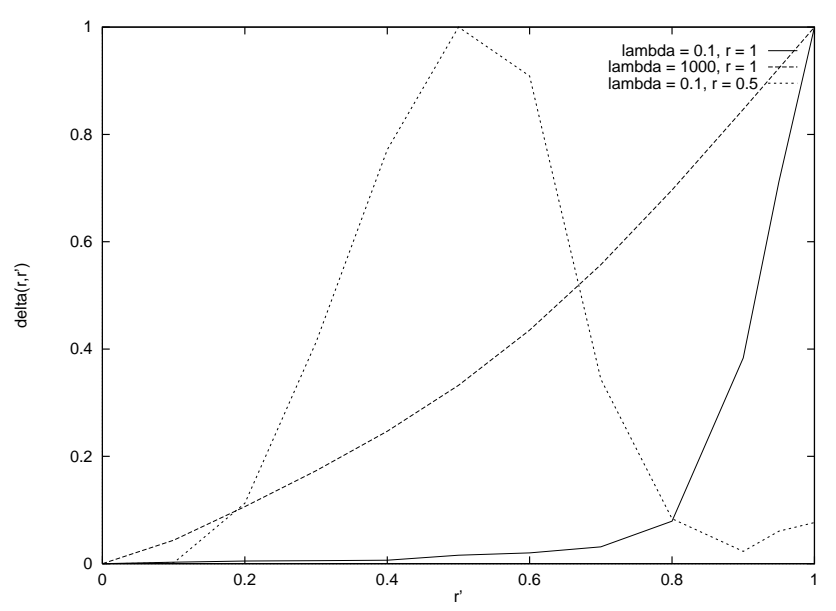

Fig. 3. The shape of the averaging kernel $\Delta\left(r, r^{\prime}\right)$ as a function of $r^{\prime}$ for three different cases. The increase in the width of $\Delta\left(r, r^{\prime}\right)$ as $\lambda$ increases is clear. Note the poor resolution at $r=0.5$, even when $\lambda$ is small

In general, one usually considers intermediate values of $\lambda$, around the turning point between the two extremes. Precisely which $\lambda$ one chooses depends on the relative importance of stability and resolution in the particular problem.

In the present case, we are interested in measuring the polarization at the limb, and it is clear from Fig. 2 that the resolution we need can only be obtained at the cost of a high standard deviation in the recovered value. If we try to increase the accuracy, we end up no longer measuring the limb polarization proper, but rather a "blurred" value of the polarisation, smoothed by convolution with the averaging kernel. If the polarization is a maximum at the limb, the bias introduced by this smoothing will reduce the estimated polarisation: the more sharply the polarization falls away from the limb, the greater this biasing will be.

Looking at the low- $\lambda$ (ie, no-noise) limit, we find that the $\Delta\left(r, r^{\prime}\right)$ function is sharply peaked at $r=1$, so that in principle we can extract a well-resolved limb-polarization. In fact, the quality of this peak degrades substantially for $r<1$ : the method as presented cannot reasonably resolve polarizations on the disk. If we wished to recover these, we might use our knowledge of the kernels and include in the sum in Eq. (7) only those kernels which are non-zero in $[0, r]$. Such a procedure would give no improvement for $r=1$.

As we increase $\lambda$, the peak broadens, but the variance of the recovered $\hat{u}_{\lambda}(r)$ decreases. In Fig. 4 we display the width $\mathcal{A}$ and variance $\mathcal{B}$ we obtain when we recover $P(1)$ using the kernel $A_{\mathrm{Q}}$, for various values of $\lambda$ from $10^{-2}$ to $10^{2}$. Here we can clearly see the trade-off between the well-resolved but unstable recovery in the bottom right corner, and the stable but badly-resolved recovery in the top left.

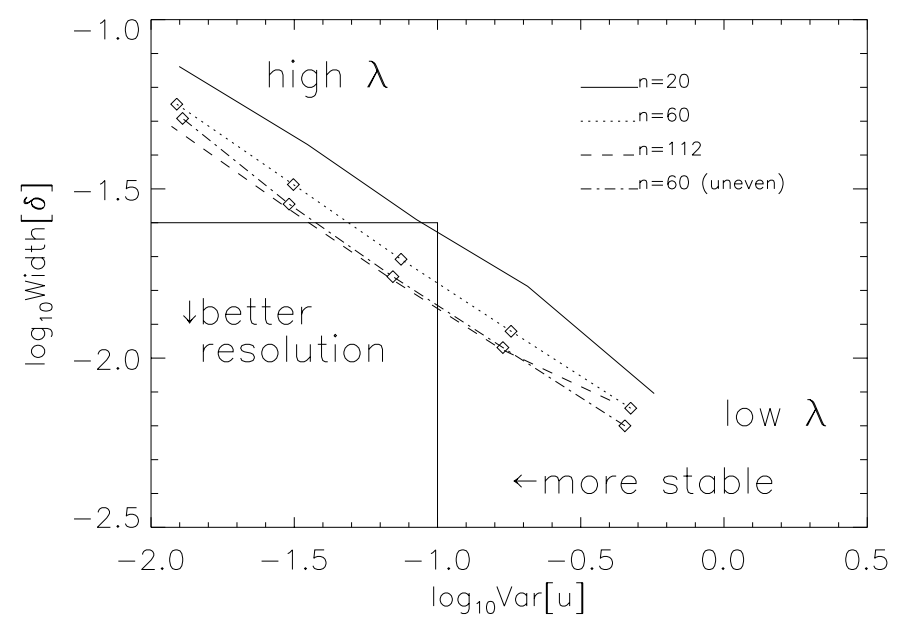

Fig. 4. The width of $\Delta\left(r, r^{\prime}\right), \log _{10} \mathcal{A}$, versus $\log _{10} \mathcal{B}=$ $\log _{10} \operatorname{Var}[\hat{u}]$, for a variety of values of $\lambda$, and a variety of sample sizes $n$. Along each line, $\log _{10} \lambda$ rises from -2 in the bottom right corner, to +2 in the top left, with integer values of $\log _{10} \lambda$ plotted with a diamond on the two $n=60$ lines. The resolution improves as we move down the plot, and becomes more stable as we move to the left; both the stability and the resolution improve as we use more data points. These curves are for $n=20$, 60,112 , as indicated; for the discussion of the line marked " $n=60$ (uneven)", see Sect. 5.3. The box in the bottom left encloses those combinations of $\lambda$ and $n$ which will produce an estimator $\hat{u}$ which is adequately stable and well-resolved

This diagram in a sense completes the Backus-Gilbert analysis of the inverse problem, as represented by the kernel in Eq. (21), and we are now in a position to move on to invert real or simulated data. Before we can do that, however, we must decide what value of $\lambda$ to use. To make that decision, we must consider the level and approximate functional form of the polarization $P(r)$, and use this to set the scale for the resolution and standard deviation we need to achieve. In turn, this fixes the number of data points $n$ we require in our data and the value of the parameter $\lambda$ we must choose in our inversion. Despite the fact that we are invoking a particular model at this point in our analysis, we emphasise that this introduces no practical model dependence. We are using an approximate model purely to help us understand what counts as "sufficiently stable" or "sufficiently well resolved", and after this understanding is gained the numbers we recover remain model independent measurements, as opposed to any method of parameter fitting.

Firstly, Chandrasekhar suggests that the limb polarization is of the order of $P(1)=0.1$; we therefore need a variance which is at least as small as this, requiring $\log _{10} \operatorname{Var}[\hat{u}]<-1$. Secondly, if we are not to have an overly biased result, our resolution function must be narrow compared with the width of the underlying function $P(r)$. The resolution we need is therefore of order Width $[P(r)]$, with the width functional used in Eq. (9). Taking $P(r) \sim \exp 10 r$ as representative, we find we 


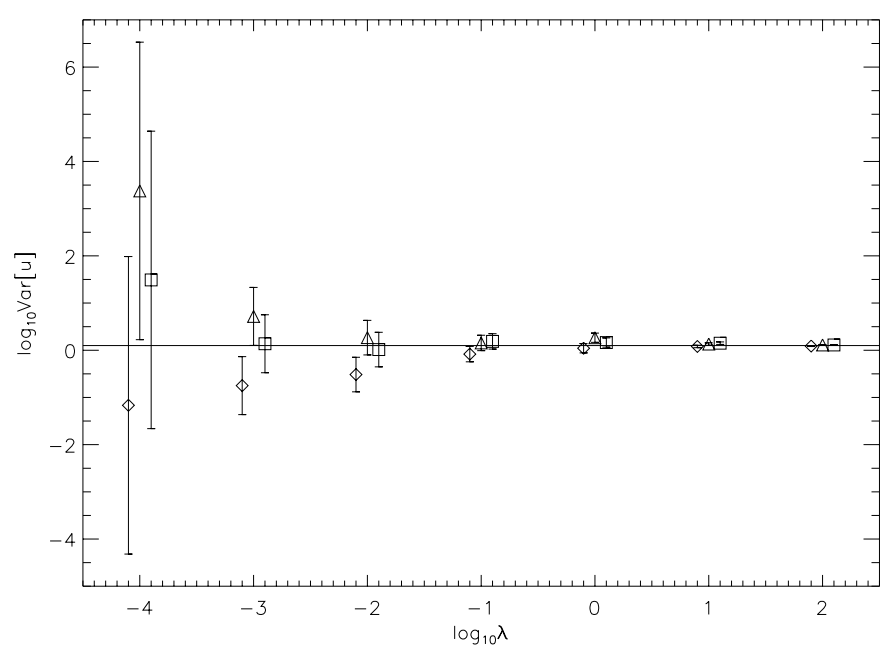

Fig. 5. The recovered values of $P(1)$ for three realisations of noisy simulated data with the same parameters. The horizontal line is the correct value of $P(1)$

need a resolution better than $\log _{10} \operatorname{Width}[\Delta]=-1.6$. Comparison with Fig. 4 indicates that $\lambda=1$ and $n \gtrsim 60$ should therefore give us a satisfactory recovery of $P(1)$. The maximum accuracy achievable for a given resolution (or vice versa) can be read off from the graph.

Figure 5 shows the recovery of the limb polarization from three sets of simulated noisy data, as a function of the smoothing parameter. It is clear that the solution becomes more stable as greater smoothing is imposed. However, there comes a point where further smoothing does nothing to improve the solution, but merely degrades the resolution of the recovery.

\subsection{The Algol system: A worked example}

As a more concrete illustration, consider the Algol system. Given the system parameters (from Söderhjelm 1980) we can analyse the observational prospects as in the previous section. The Algol system is more complex than the spherically symmetric cases we consider, but our simplified analysis will provide an upper bound on the resolution achievable with the real system.

The graphs presented by Kemp et al. (1983) have 25 data points in the eclipse phase. The same paper states that, in the Algol primary star, $50 \%$ of the polarized flux comes from an annulus on the limb of width less than $0.005 R_{\text {disc }}$.

Our Backus-Gilbert analysis of a spherically symmetric model system with the Algol orbital and luminosity parameters indicates that this limb polarization profile cannot be resolved in the Algol system. For the eclipse coverage reported in Kemp et al. (1983) the minimum width $\mathcal{A}_{\text {min }}$ of the averaging kernel at the limb is $0.0199 R_{\text {disc }}$, even in the zero-noise limit.

This indicates that the limb polarization cannot be truly resolved with this data if the polarization profile is really as sharp as the stellar models cited by Kemp et al. predict. Thus, while the data does indicate the detection of limb polarization, it cannot reasonably be used (as was attempted by Wilson \& Liou 1993) to make a quantitative estimate of the polarization profile.

The precise dependence of the resolution on the number of data points is beyond the scope of the present paper, but we note here that increasing the number of points from 25 to as many as 1000 does not reduce the kernel width sufficiently (from $0.019 R_{\text {disc }}$ to $0.0144 R_{\text {disc }}$ ). For practical purposes, then, the inadequate resolution is intrinsic to the Algol system and will not be alleviated by improved observations.

\subsection{Observational strategies}

Given a set of measurements $f_{i}$ at positions $s_{i}$, the Backus-Gilbert method can give us a well-controlled recovery of the underlying function $u(r)$. We can do better than this, however, as the method can suggest how to improve our observational strategy to improve the resolution of the recovery. Clearly, increasing the total number of measurements we take, or improving the noise on each measurement, will improve the quality of our recovery. Equally clearly, simply binning the data (so that $\sigma \sim \sqrt{n}$ ) demonstrably improves nothing (this can be thought of as conservation of information).

Even if we assume, however, that we cannot change the number or quality of our data points (telescope time and equipment are limited, after all), we might still have the freedom to adjust the positions $s_{i}$ (i.e., the times) at which me make our measurements.

In Eq. (7), we are averaging the data points $f_{i}$ with a weight vector $q_{i}$. Where $q_{i}$ is relatively large, therefore, $f_{i}$ will be smeared over several $q_{i}$, or over a range of $s_{i}$, smearing out features in the kernel $K\left(r ; s_{i}\right)$. If we can identify prominent features in the vector $q_{i}$, and cluster our measurements round the $s_{i}$ they correspond to, we should be able to decrease the spread of $\Delta\left(r, r^{\prime}\right)$ and so increase the resolution of the recovery.

Our simulations show that this rather informal argument is valid for our case. The line in Fig. 4 captioned " $n=60$ (uneven)" has the same number of data points as the line " $n=60$ ", but with the values $s_{i}$ chosen so that the "data rate" in a band $s=1.85 \pm 0.15$ is double that outside the band. This does not significantly improve the variance of the result (we are not gathering any more information than before), but it does noticeably improve its resolution.

This improvement in our procedure corresponds to concentrating our measurements on the point around the beginning of the eclipse. We might have guessed that this would be a reasonable strategy to adopt, but the BackusGilbert method has justified our guess, and would substitute for a lack of intuition in a more obscure situation. 


\section{Conclusions}

Our studies of simulated data show the fundamental limitations on the determination of limb polarization in eclipsing binary stars. In particular, Fig. 4 indicates that limb polarizations of order a few percent are only just above the threshold of detectability, even in perfectly spherically symmetric, non-interacting binary systems. The situation will be worse in more complex systems.

It is important to appreciate that the Backus-Gilbert method does not strictly estimate the polarization at a point on the stellar disc, but rather the polarization convolved with the resolution function. To relate the results of the inversion to a particular model, it is necessary to calculate the theoretical value of this convolution, which should be consistent with the $\lambda$ we have chosen and with all higher values of $\lambda$ - these represent coarser averages over the stellar disc.

The bottom line is that one must take care in drawing conclusions about limb polarization from studies of eclipsing binaries. It is clearly not possible to distinguish between stellar atmosphere models on this basis if their predicted limb polarizations differ by less than the maximum accuracy achievable. On the other hand, an appreciation of the issues raised in this paper will allow a meaningful determination of limb polarization, with reliable error estimates.

Formally, the eclipsing binary problem is very similar to the gravitational microlensing problem. Indeed, part of the initial motivation for this work sprang from studies of the microlensing of extended sources. A future paper (Coleman et al. in preparation) will apply the inverse problem approach outlined in this paper to the use of microlensing as a probe of stellar atmospheres.

Acknowledgements. We would like to thank Dr. Richard K. Barrett for enlightening discussions of the Backus-Gilbert method. IJC was supported by a PPARC studentship.

\section{Appendix A: General derivation of the Backus-Gilbert index}

In Sect. 3, we described the B-G inverse in rather practical terms, taking care to relate the description to the quantities obtained in, and the concerns relevant to, real observations. Such a physical understanding of the method is essential if it is to be used properly, but we can obtain other insights into the method by reexamining it in a more formal way ${ }^{1}$.

Equation (4) above describes an operator $K: P \rightarrow D$ mapping an object from a source space $P$ into a data space $D$. Including noise $n \in D$, we have

$f=K u+n$

\footnotetext{
${ }^{1}$ We thank the referee, Dr. A Lannes, for suggesting this
} approach. for $f \in D$ and $u \in P$. Here $P$ is a real Hilbert space, parametrised by $r$, with a symmetric inner product

$(a \mid b)_{P}=\int_{0}^{1} a(r) b(r) \mathrm{d} r \quad, \forall a, b \in P$

and $D$ is a finite-dimensional Euclidean space with

$(a \mid b)_{D}=\sum_{i} a_{i} b_{i}$

We wish to make an estimate $\hat{u}_{r} \in \mathbf{R}$ of a single component of the object $u$, based on the data $f$. To this end, we wish to find a $q \in D$ (depending on $r$ ), such that

$\hat{u}_{r}=(q \mid f)_{D}$.

We find this $q$ as the solution of a minimisation problem. Introducing the adjoint operator $K^{*}: D \rightarrow P$, and assuming $E\left((q \mid n)_{D}\right)=0$, we have

$E\left(\hat{u}_{r}\right)=(q \mid K u)_{D}=\left(K^{*} q \mid u\right)_{P}$.

Recalling that $\left(K^{*} q \mid u\right)_{P}=\int_{0}^{1}\left(K^{*} q\right)\left(r^{\prime}\right) u\left(r^{\prime}\right) \mathrm{d} r^{\prime}$, we see that $\left(K^{*} q\right)\left(r^{\prime}\right) \in P$ can be identified with the averaging kernel $\Delta\left(r, r^{\prime}\right)$, and that Eq. (A3) will be a good estimate of $u_{r}$ when $q$ is transformed by $K^{*}$ into the basis vector $e_{r} \in P$ corresponding to the component $r$ of $u$. That is, Eq. (A3) would be exact if $K^{*} q=e_{r}$. The object $K^{*} q$ will instead be a linear combination of basis vectors "close" to $e_{r}$, and we can measure its "scatter' around $e_{r}$ with the operator $Q: P \rightarrow P$ such that $Q x_{r^{\prime}} \equiv\left(r-r^{\prime}\right)^{2} x_{r^{\prime}}, \forall x_{r^{\prime}} \in$ $P$. Define

$\mathcal{A} \equiv\left(K^{*} q \mid Q K^{*} q\right)_{P}=\left(q \mid K Q K^{*} q\right)_{D}=\sum_{i, j} q_{i} W_{i j} q_{j}$,

defining the (self-adjoint) operator $W=K Q K^{*}: D \rightarrow D$. We may also define a measure of the stability of $\hat{u}_{r}$,

$\mathcal{B} \equiv(q \mid S q)_{D}$

by analogy with Eq. (11), where the operator $S \in D$ is such that $\left(e_{i} \mid S e_{j}\right)_{D}=S_{i j}$, where $S_{i j}$ is the positive definite noise covariance matrix. The demand that $\Delta\left(r, r^{\prime}\right)$ have unit area translates into the constraint $\left(K^{*} q \mid 1\right)_{P}=1$, where $1 \in P$ is the all- 1 vector in $P$. Writing $R \equiv K 1 \in D$, this is equivalent to the constraint

$(q \mid R)_{D}=1$,

restricting $q$ to a hypersurface in $D$, with normal $R$.

If we now introduce the functional $c: D \rightarrow \mathbf{R}$, such that

$c(q) \equiv \frac{1}{2}[\mathcal{A}(q)+\lambda \mathcal{B}(q)]=\frac{1}{2}(q \mid(W+\lambda S) q)_{D}$,

the minimisation problem becomes that of finding the $q$ which minimises $c(q)$, subject to $(q \mid R)_{D}=1$. Considering small variations $\epsilon \in D$ in the hyperplane (that is $\{\epsilon$ : $\left.(\epsilon \mid R)_{D}=0\right\}$, and defining the gradient $\nabla c(q)=(W+\lambda S) q$ ( $W+\lambda S$ is self-adjoint since both $W$ and $S$ are), we have $c(q+\epsilon)=c(q)+(\epsilon \mid \nabla c(q))_{D}+\frac{1}{2}(\epsilon \mid(W+\lambda S) \epsilon)_{D}$.

This is extremised at $q_{\lambda}$ such that $\left(\epsilon \mid \nabla c\left(q_{\lambda}\right)\right)_{D}=0$, and is a minimum if $(\epsilon \mid(W+\lambda S) \epsilon)_{D} \geq 0$. The operator $S$ is positive-definite by definition, and the operator $W$ is 
positive-definite, since $Q$ is. The operator $c(q)$ is therefore minimised when $\left(\epsilon \mid(W+\lambda S) q_{\lambda}\right)_{D}=0, \forall \epsilon$, or

$(W+\lambda S) q_{\lambda}=\alpha R$,

for any $\alpha \in \mathbf{R}$. Imposing the constraint Eq. (A6), we thus find

$q_{\lambda}=\frac{(W+\lambda S)^{-1} R}{\left(R \mid(W+\lambda S)^{-1) R} D\right.}$,

from which we can obtain Eq. (13), from the definition of $(\cdot \mid \cdot)_{D}$

\section{References}

Backus G., Gilbert F., 1970, Phil. Trans. R. Soc. London 266, 123

Chandrasekhar S., 1946, ApJ 103, 351

Chandrasekhar S., 1950, Radiative Transfer. Clarendon Press, Oxford
Collins G.W.II, Buerger, P.F., Polarization from illuminated nongray stellar atmospheres, in Planets, Stars and Nebulae, Gehrels T. (ed.). Univ. Arizona Press, Tucson p. 663

Clarke D., Grainger J.F., 1971, Polarized Light and Optical Measurement. Pergamon Press

Coleman I.J., Gray N., Simmons J.F.L., Inversion of microlensing light curves from finite sources (in preparation)

Craig I.J.D, Brown J.C., 1986, Inverse Problems In Astronomy. Adam Hilger

Kemp J.C, Henson G.D., Barbour M.S., Kraus D.J., 1983, ApJ 273, L85

Landi Degl'Innocenti E., Landi Degl'Innocenti M., Landolfi M., 1988, A\&A 204, 133

Loredo T.J., Epstein R.I., 1989, ApJ 336, 896

Parker R.L., 1977, Ann. Rev. Earth Planet. Sci. 5, 35

Simmons J.F.L., Newsam A.M., Willis J.P., 1995, MNRAS 276, 182

Söderhjelm S., 1980, A\&A 89, 100

Wilson R.E., Liou J.C., 1993, ApJ 413, 670 